\title{
Bridging the nucleation step - the link of molecular interactions in dilute solutions and the crystal structure
}

\author{
K. Edkins \\ School of Health Sciences, University of Manchester, Oxford Road, Manchester M13 9PL, United Kingdom, \\ Katharina.edkins@manchester.ac.uk
}

The solvent influence on crystallisation outcome has been shown in a large number of cases, most often as the observation of different crystal forms crystallising from recrystallization from different solvents. More detailed work has been conducted to investigate solutesolute and solute-solvent interaction in solution with increasing saturation to mimic the crystallisation process, and to understand and use the solvent influence on crystallisation with the ultimate aim to control the crystallisation outcome.[1, 2, 3] However, to date there are contradicting opinions whether solution interaction drives the nucleation of a particular crystal form or if other factors such as the exact nucleation pathway, solvation state of clusters and solute conformations, outweigh the solvent influence.[4]

But can the nucleation step be completely ignored? Our hypothesis is that strong intermolecular interactions in dilute solution are likely to be carried through the nucleation step into the final crystal structure independent from the nucleation pathway followed, and weak interactions are unlikely to survive the nucleation step. Verification of this hypothesis would allow us to directly connect dilute solutions with the crystallisation product, and even allow for prediction of the existence of a particular crystal form before performing crystallisation experiments.

Using a combination of vibrational and nuclear magnetic spectroscopy, X-ray and neutron diffraction and molecular dynamics simulations, I will show the link between solution and solid-state interactions for multi-component crystal forms and how the microscopic structure of the solution can influence the crystallisation outcome.[5]

[1] Davey, R. J., Dent, G., Mughal, R. K., Parveen, S. (2006). Cryst. Growth Des. 6, 1788.

[2] Hunter, C. A., McCabe, J. F., Spitaleri, A. (2012). CrystEngComm 14, 7115.

[3] Derdour, L., Skliar, D. (2014). Chem. Eng. Sci. 106, 275.

[4] Du, W., Cruz-Cabeza, A. J., Woutersen, S., Davey, R. J., Yin, Q. (2015). Chem. Sci. 6, 3515.

[5] Jones, C. D., Walker, M., Xiao, Y., Edkins, K. (2019). Chem. Commun. 55, 4865.

Keywords: Pre-nucleation aggregation; pharmaceuticals; multi-component 